\title{
Gastric follicular lymphoma: A report of 3 cases and a review of the literature
}

\author{
HEE YOUNG NA ${ }^{1 *}$, YOUNG A KIM $^{2,3 *}$, CHEOL LEE $^{2}$, JI-YOUNG CHOE $^{2,4}$, SEON AH SHIN $^{2}$, \\ JUNG-WEON SHIM ${ }^{5}$, SOO KEE MIN ${ }^{4}$, HYUN-JUNG KIM ${ }^{6}$, JAE HO HAN ${ }^{7}$ and JI EUN KIM ${ }^{2,3}$ \\ ${ }^{1}$ Department of Pathology, Seoul National University Bundang Hospital, Seongnam, Gyeonggi 13620; \\ ${ }^{2}$ Department of Pathology, College of Medicine, Seoul National University, Seoul 03080; ${ }^{3}$ Department of Pathology, \\ Seoul National University Boramae Hospital, Seoul 07061; ${ }^{4}$ Department of Pathology, Hallym University Sacred Heart \\ Hospital, Anyang, Gyeonggi 14068; ${ }^{5}$ Department of Pathology, Dongtan Sacred Heart Hospital Hallym University, \\ Dongtan, Gyeonggi 18450; ${ }^{6}$ Department of Pathology, Inje University Sanggye Paik Hospital, Seoul 01757; \\ ${ }^{7}$ Department of Pathology, Ajou University School of Medicine, Suwon, Gyeonggi 03965, Republic of Korea
}

Received August 3, 2016; Accepted February 20, 2018

DOI: $10.3892 / \mathrm{ol} .2018 .8744$

\begin{abstract}
Follicular lymphoma (FL) occurs primarily in the gastrointestinal tract, with the stomach being one of the rarest sites. According to the literature, $<20$ cases of primary gastric FL have been reported, with the number of cases with detailed pathological descriptions being even less. The aim of the present study was to compare clinicopathological features of gastric FL with FL at alternative sites. A total of 3 cases of gastric FL were retrieved from among 3,216 cases in the databases of 4 university hospitals in South Korea: Seoul National University (SNU) Hospital, SNU Boramae Hospital, SNU Bundang Hospital (all Seoul, South Korea) and Dongtan Sacred Heart Hospital Hallym University (Dongtan, South Korea), including 2 primary cases and 1 case that was possibly secondary to nodal FL. The 2 primary gastric FL cases were incidentally detected in routine health check-ups. An endoscopy revealed a single polypoid submucosal mass and biopsies failed to confirm the diagnosis due to minimal mucosal involvement. Therefore, a partial gastrectomy was performed. The epicenters of the tumors were submucosal, with focal extension to the muscularis propria. However, 1 case exhibited an isolated FL nodule in the omentum. Histopathological examination revealed FL of grade 1-2 with a follicular pattern and with strong expression of germinal center markers and B-cell lymphoma 2 (BCL2). Rearrangement of BCL2 was not identified using fluorescence in situ hybridization
\end{abstract}

Correspondence to: Dr Ji Eun Kim, Department of Pathology, Seoul National University Boramae Hospital, 20 Boramae-Ro 5-Gil, Dongjak, Seoul 07061, Republic of Korea

E-mail: npol1181@snu.ac.kr

${ }^{*}$ Contributed equally

Key words: follicular lymphoma, gastric lymphoma, B-cell lymphoma 2 studies in 2 cases. In contrast to these 2 cases, the remaining FL case was confirmed with an endoscopic biopsy. The endoscopy revealed multiple eroded polypoid lesions, and pathology revealed FL of grade 1-2 with a predominantly diffuse pattern, and with immunoglobulin heavy chain IGH/BCL2 translocation. In view of the extensive lymphadenopathy, the last case possibly presented as secondary involvement of nodal FL. It is challenging to diagnose FL in the stomach due to little mucosal involvement, as well as the unfamiliarity of the tumor due to its rarity. However, the results of the present study suggest that primary gastric FL may exhibit unique pathological features, including a predominantly follicular pattern and an absence of BCL2 rearrangement.

\section{Introduction}

The stomach is the most frequent site of malignant lymphoma involving the gastrointestinal (GI) tract. Among the subtypes of malignant lymphoma, extranodal marginal zone lymphoma of mucosa-associated lymphoid tissue (MALT lymphoma) and diffuse large B-cell lymphoma (DLBCL) account for $>90 \%$ of all cases (1). The incidence of Follicular lymphoma (FL), whether primary or otherwise, has been reported to account for between 0.8 and $3 \%$ of gastric lymphomas (2). FL is one of the most common types of non-Hodgkin lymphoma in western countries including United States of America (3) and United Kingdom (4), whereas the incidence in Asian countries including Korea (5), Japan (6) and China (7) is lower than that of western countries. The incidence of FL is typically low in Korea, occupying $2.9 \%$ of malignant lymphoma (8).

Histologic features of FL are characterized by nodular aggregation of two different types of tumor cells, which are small cleaved centrocytes and large centroblasts, and WHO histologic grading system is based on the number of centroblasts per high power field (9). Clinically FL commonly presents as widely spread systemic disease, and prognosis varies according to the extent of the disease and histologic grading at the time of diagnosis (10). In localized early stage 
diseases, watchful waiting or radiotherapy is available due to its slowly progressive nature, while chemotherapy including rituximab is selected in advanced stage diseases with high tumor burdens (11). The origin of tumor cells is germinal center B-cells which are heavily crowded in lymphoid organs, especially lymph nodes (9). Therefore, FL has been recognized as primary nodal lymphoma, and primary extranodal FL has been described in only a few organs including the skin and duodenum (12). The second part of the duodenum is known to be the most frequent site of FL of the GI tract followed by jejunum (10). In the latest revised fourth edition of WHO classification, duodenal FL was descried as a distinct variant of FL with typical clinical and immunophenotypical characteristics. However, in majority of cases, FL of the GI tract may present through secondary involvement by widespread nodal FL, therefore, primary gastric FL appears to be rare $(9,10,12,13-17)$. The present study describes 3 cases of gastric FL alongside a comprehensive review of previous cases reported in the literature and compares their features with those of duodenal FL.

\section{Case report}

Case selection. A total of 3 cases of gastric FL were retrieved from among the cases in the databases of 4 university hospitals in South Korea (SNU Hospital, SNU Boramae Hospital, SNU Bundang Hospital and Dongtan Sacred Heart Hospital Hallym University). The cases were selected from a total of 3,216 cases of non-Hodgkin's lymphoma of the GI tract, occurring between January 2000 and December 2015, as the only 3 cases of gastric FL. The pathological features were reviewed by 3 experienced hematopathologists. Due to the retrospective nature of the study, ethical approval and patient consent for publication was waived for the present study by the Institutional Review Board of Seoul National University Boramae Hospital (Seoul, South Korea).

Case 1. A 61-year-old man was referred to SNU Boramae Hospital in November, 2015 for the management of a gastric lesion detected during an annual health check-up program at a local clinic. Endoscopic examination revealed a well demarcated, polypoid mass in the anterior wall of the gastric body. Despite three repeat biopsies, the preoperative pathological results remained unclear, simply suggesting a malignant lymphoma of germinal center B-cell origin. Constitutional symptoms, including lymphadenopathy, organomegaly and anemia, were not exhibited. A partial gastrectomy was performed and revealed a well demarcated, protruded mass, measuring $2.0 \times 1.8-\mathrm{cm}$ in the body, as well as an additional $1.5 \times 1.2-\mathrm{cm}$ nodule in the lesser omentum. Specimens were fixed using $10 \%$ formaldehyde for $24 \mathrm{~h}$ at room temperature and embedded in paraffin at room temperature for $24 \mathrm{~h}$. Serial 4- $\mu$ m-thick sections were sliced and were stained with $0.5 \%$ hematoxylin staining solution for $20 \mathrm{~min}$ at room temperature. Subsequently, $1 \%$ hydrochloric acid alcohol was added for $1 \mathrm{~min}$. The slides were stained with $1 \%$ eosin Y staining solution for $1 \mathrm{~min}$ at room temperature and dehydrated at room temperature after applying tap water at room temperature for $15 \mathrm{~min}$. and immunohistochemistry (IHC) for CD3 (clone F7.2.38, Dako; Agilent Technologies, Inc., Santa Clara, CA,
USA; 1:600), CD5 (clone 4C7, DAKO; 1:100), CD20 (clone L26, Dako; Agilent Technologies, Inc: 1:200), BCL2 (clone 124, DAKO; Agilent Technologies, Inc.; 1:50), BCL6 (clone LN22, Leica Microsystems, Ltd., Milton Keynes, UK; 1:60), CD10 (clone 56C6, Leica Microsystems, Ltd; 1:30), CD23 (clone SP23, Thermo Fisher Scientific, Inc., Waltham, MA, USA; 1:100), CD43 (clone DF-T1, DAKO; 1:2,500), cyclin D1 (clone SP4, Thermo Fisher Scientific, Inc: 1:25) and MUM1 (clone EAU32, Leica Microsystems, Ltd; 1:100). IHC staining was performed using the Ventana Benchmark XT system (Ventana Medical Systems, Inc., Tucson, AZ, USA) or a Bond-Max automated immunostainer (Leica Microsystems, Ltd.). Sections were dried in oven at $60^{\circ} \mathrm{C}$ for $30 \mathrm{~min}$ and deparaffinized in xylene and rehydrated through a series of graded ethanol solutions $(95,85,70$ and $55 \%$ ) at room temperature for $10 \mathrm{~min}$. Antigen retrieval was performed in a pressure cooker at $95^{\circ} \mathrm{C}$ for 2 min using $0.01 \mathrm{M}$ citrate buffer ( $\mathrm{pH} \mathrm{6.0)}$ and incubated overnight at $4^{\circ} \mathrm{C}$ for all the primary antibodies. Then the slides were washed by phosphate buffered saline (PBS) four times. After warming to $37^{\circ} \mathrm{C}$, detection involved ultraView Universal DAB Detection kit (cat. 760-500, Ventana Medical Systems, Inc.) that includes a cocktail of horseradish peroxidase-labeled goat anti-rabbit, mouse $\operatorname{IgG}$ secondary antibodies for $16 \mathrm{~min}$. The complex was then visualized with 3,3'-diaminobenzidine tetrahydrochloride (DAB) chromogen according to the manufacturer's protocol. The samples were then analyzed by a BX51 light microscope (magnification, x200 and x400) (Olympus Corporation, Tokyo, Japan). Histologically, the two tumors comprised small-to medium-sized lymphoid cells with complete nodular architecture. Lymphoepithelial lesions and monocytoid or plasmacytoid features indicative of MALT lymphoma were not identified. The epicenter of the tumor was the submucosa, however, the tumor extended to the muscularis propria, with only focal involvement of the mucosa. Immunohistochemistry (IHC) indicated that the tumor cells were positive for cluster of differentiation CD10, CD20, B-cell lymphoma BCL2 and BCL6, and negative for CD3, CD5, CD23, CD43, cyclin D1 and mutated melanoma-associated antigen (MUM) 1/interferon regulatory factor (IRF)4. Follicular dendritic cell (FDC) networks were preserved within the CD21-positive nodules, demonstrating a predominantly compact pattern with focal abolishment at the center of the nodule (Fig. 1). The patient was diagnosed with FL of grade 1-2 with a follicular pattern according to the WHO classification (10). A fluorescence in situ hybridization (FISH) test for locus-specific identifier immunoglobulin heavy chain (IGH)/BCL2 dual fusion translocation probe (Vysis; Abbott Pharmaceutical Co., Ltd., Lake Bluff, IL, USA) and BCL2 break-apart probe (Vysis; Abbott Pharmaceutical Co., Ltd.) revealed no rearrangement of BCL2 at 18q21 (18). The background gastric mucosa demonstrated prominent lymphoid follicles with Helicobacter pylori (H. pylori) colonization. As the omental tumor was interpreted as a multiplicity rather than a metastatic deposit, the patient's final clinical stage was IE2 using the modified Ann Arbor $(19,20)$ staging system, or I using the Lugano (21) staging system. The patient was treated with 6 cycles of chemotherapy [intravenous (IV) rituximab $375 \mathrm{mg} / \mathrm{m}^{2}$, cyclophosphamide $750 \mathrm{mg} / \mathrm{m}^{2} \mathrm{IV}$, vincristine $1.4 \mathrm{mg} / \mathrm{m}^{2} \mathrm{IV}$ and prednisolone $40 \mathrm{mg} / \mathrm{m}^{2}$ per oral (PO), R-CVP] every 21 days. The patient came to hospital 

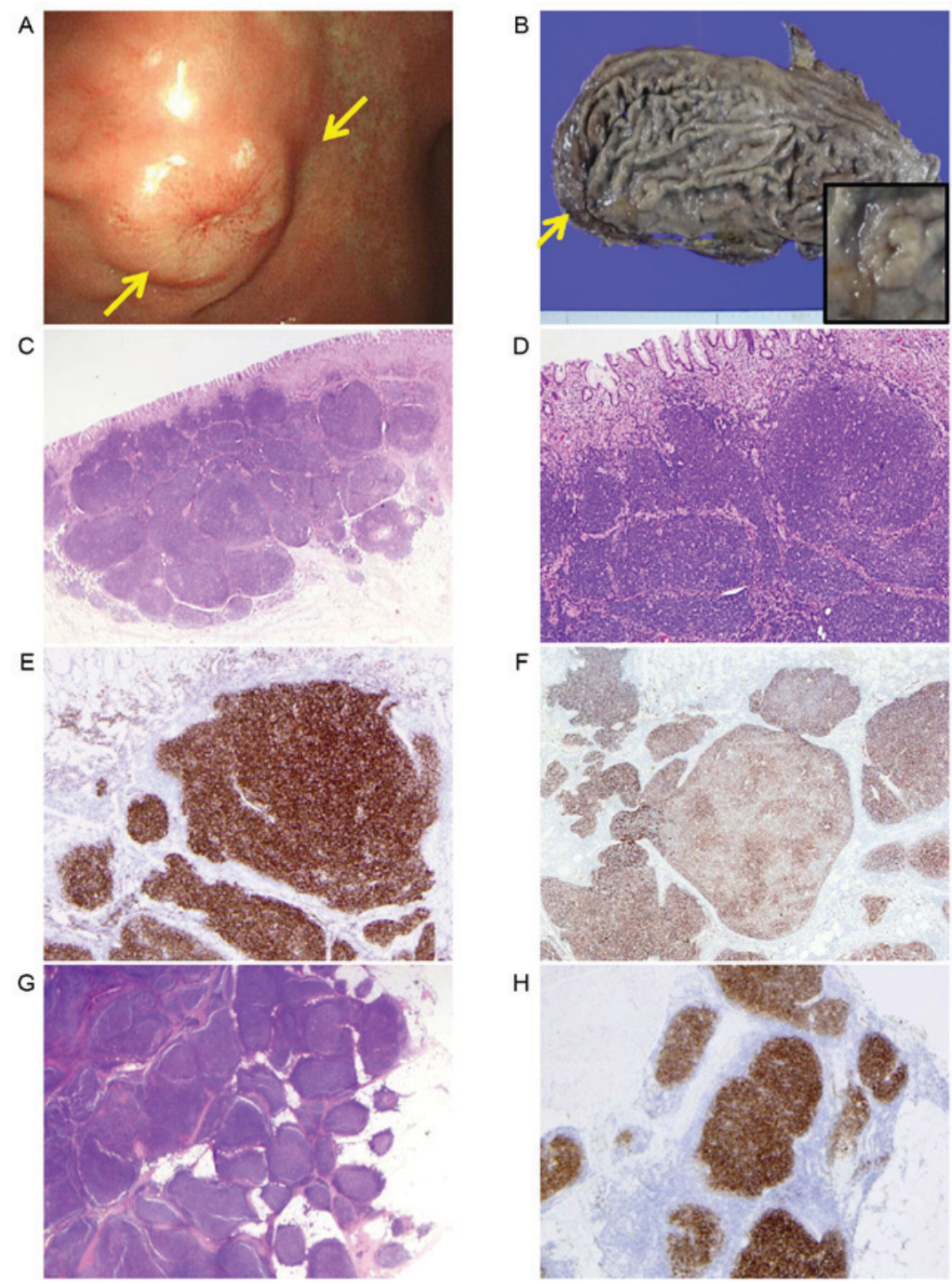

Figure 1. Representative features of case 1, primary gastric follicular lymphoma. (A) An endoscopy and (B) the resected specimen revealed a well demarcated polypoid mass in the gastric body. Microscopically, nodular aggregates of small lymphoid cells in the submucosa with little mucosal involvement are characteristic, as demonstrated by hematoxylin and eosin staining at (C) x10 and (D) magnification, x40. (E) Tumor cells were positive for CD10 (magnification, x40). (F) The follicular dendritic cell network was preserved, using anti-CD21 (magnification, $\mathrm{x} 40$ ). The omental nodule revealed similar results following (G) hematoxylin and eosin and (H) anti-CD10 staining (magnification, $\mathrm{x} 40$ ). CD, cluster of differentiation.

every month and remained disease-free at the last follow-up (13 months following diagnosis).

Case 2. A 50-year-old man underwent gastroscopy for routine health check-up in Dongtan Sacred Heart Hospital and presented with a gastric lesion; a single elevated submucosal mass at the fundus, grossly indicative of a GI stromal tumor. As in the first case, no additional abnormalities were identified through systemic examination. The patient underwent a partial gastrectomy following an endoscopic biopsy that failed to provide a pathological diagnosis, which revealed a $1.8 \times 1.6-\mathrm{cm}$ submucosal tumor extending to the muscularis propria. The microscopic features and IHC results were near identical to those in case 1, with the exception of the absence of separate tumor nodule in the omentum. Rearrangement of BCL2 was not detected using the IGH/BCL2 dual fusion probe or BCL2 break-apart probe by FISH (Fig. 2). The colonization of $H$.pylori was not identified in the surrounding gastric mucosa.
The patient's final stage was IE2 using the modified Ann Arbor staging system $(19,20)$ and stage I using the Lugano staging system (21). The patient refused any further treatment and remained disease-free for 30 months following the surgery. Subsequently, the patient was lost to but follow-up.

Case 3. A 40-year-old man presented with epigastric discomfort for several months. An endoscopy performed on December, 2015 at Seoul National University Hospital (Seoul, Korea) revealed multiple elevated mass-like lesions at the mid-body of the stomach, on the lesser curvature side, and a biopsy confirmed FL of grade 1-2 with a predominantly diffuse pattern. Unlike the 2 aforementioned cases, the subepithelial lamina propria was densely filled with small-to medium-sized lymphoid cells without nodular aggregation. Despite extensive lymphoid infiltration, definite lymphoepithelial lesions were not identified. The tumor cells were positive for CD10, CD20, BCL2 and BCL6, and negative 

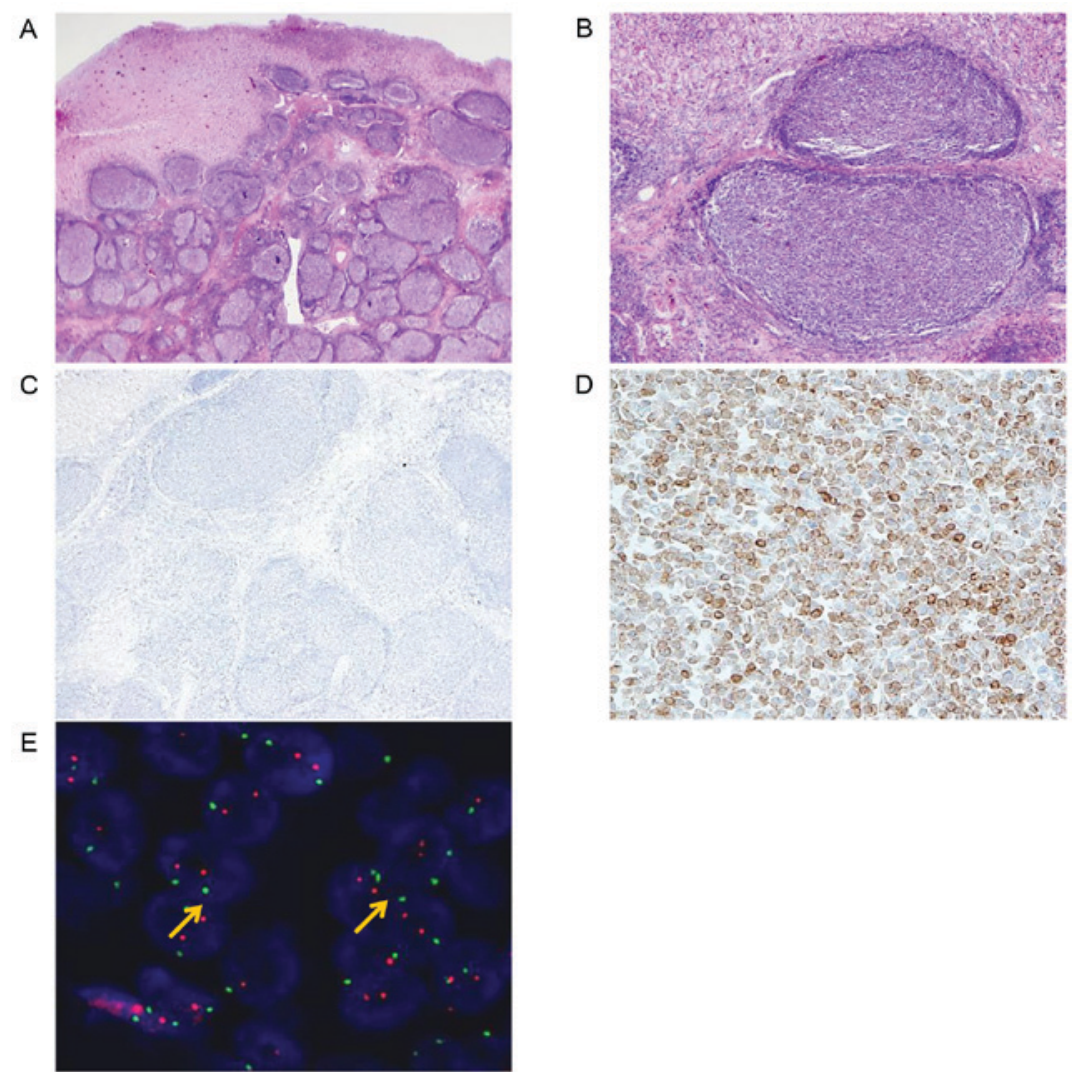

Figure 2. Representative features of case 2, primary gastric follicular lymphoma. Microscopic results identified follicular lymphoma centered in the submucosa at (A) x10 and (B) x40 magnification using hematoxylin and eosin staining. (C) No follicular dendritic cells were identified using anti-cluster of differentiation 21 (magnification, x40). (D) Tumor cells expressed BCL2 protein (magnification, x400), however, (E) the majority of tumor cells exhibited two, split, red and green signals in fluorescence in situ hybridization using a dual fusion probe. BCL2, B-cell lymphoma 2.

for CD3, cyclin D1 and MUM1/IRF4, according to the IHC results. The tumor cells were also positive for IGH/BCL2 translocation, as confirmed by FISH (Fig. 3). In addition to the gastric lesion, multiple large hypermetabolic lymph nodes $(\leq 12.0 \mathrm{~cm})$ were identified in the retroperitoneum, mesentery and abdomen. A bone marrow examination revealed the involvement of FL. The patient's final stage was IVA using the Ann Arbor staging system $(10,18)$. It was concluded that the gastric lesion was possibly secondary to nodal FL, due to the presence of massive lymphadenopathy. The patient underwent 6 cycles of R-CVP (rituximab $375 \mathrm{mg} / \mathrm{m}^{2} \mathrm{IV}$, cyclophosphamide $750 \mathrm{mg} / \mathrm{m}^{2} \mathrm{IV}$, vincristine $1.4 \mathrm{mg} / \mathrm{m}^{2} \mathrm{IV}$ and prednisolone $40 \mathrm{mg} / \mathrm{m}^{2} \mathrm{PO}$ ) every 21 days without surgical intervention, demonstrating partial response on computed tomography (CT) scan. Following R-CVP, he received 9 cycles of rituximab maintenance therapy $\left(750 \mathrm{mg} / \mathrm{m}^{2}\right.$ IV every 2 months) and showed complete response. At time of publication, he is planning to undergo 3 more cycles of maintenance chemotherapy (26 months post-diagnosis).

\section{Discussion}

FL is a basic nodal-based neoplasm, although several cases of primary extranodal FL have previously been described as specific variants $(10,12,13-15)$. Involvement of the GI tract by advanced nodal FL may be uncommon, although accurate incidence rates have not been reported (22). Among extranodal FL, primary gastric FL is rare. According to a nationwide survey of malignant lymphoma among Koreans released in 2011 (8), there was no single case of gastric FL in the 1,112 recorded cases of GI lymphoma. To date, $<20$ cases of primary gastric FL with well documented pathological results have been reported in the literature, although the overall incidence should be slightly higher (Table I) $(13,16,23-25)$. Due to the higher incidence of MALT lymphoma in stomach, and the similar cytologic morphology of tumor cells of MALT lymphoma and FL, primary gastric FL may have been misdiagnosed as MALT lymphoma, especially in small biopsy specimens. Based on a review of previous studies, the majority of gastric FL presented as single- or multiple-polypoid submucosal masses, which could hinder the accurate diagnosis through endoscopic mucosal biopsy. Histologically, the tumor cells were of low grade, consistent with grade 1-2 and exhibited a predominantly follicular pattern, similar to those observed in 2 of the cases described in the present study. In contrast to cases 1 and 2, case 3 was confirmed as FL by endoscopic biopsy due to diffuse tumor cell infiltration in the lamina propria. This diffuse mucosal involvement may be a useful feature to differentiate secondary involvement by nodal FL from primary gastric FL. Takata et al $(13,14)$ suggested that an FDC pattern can help differentiate primary duodenal FL from nodal FL by performing IHC for FDC markers (CD23 or CD43); loss of FDC network in the center, with remnant peripheral FDC exhibiting a 'hollow pattern' is identified in duodenal FL, whereas a compact nodular network of FDCs is exhibited in nodal FL. The FDCs are reported to provide a 

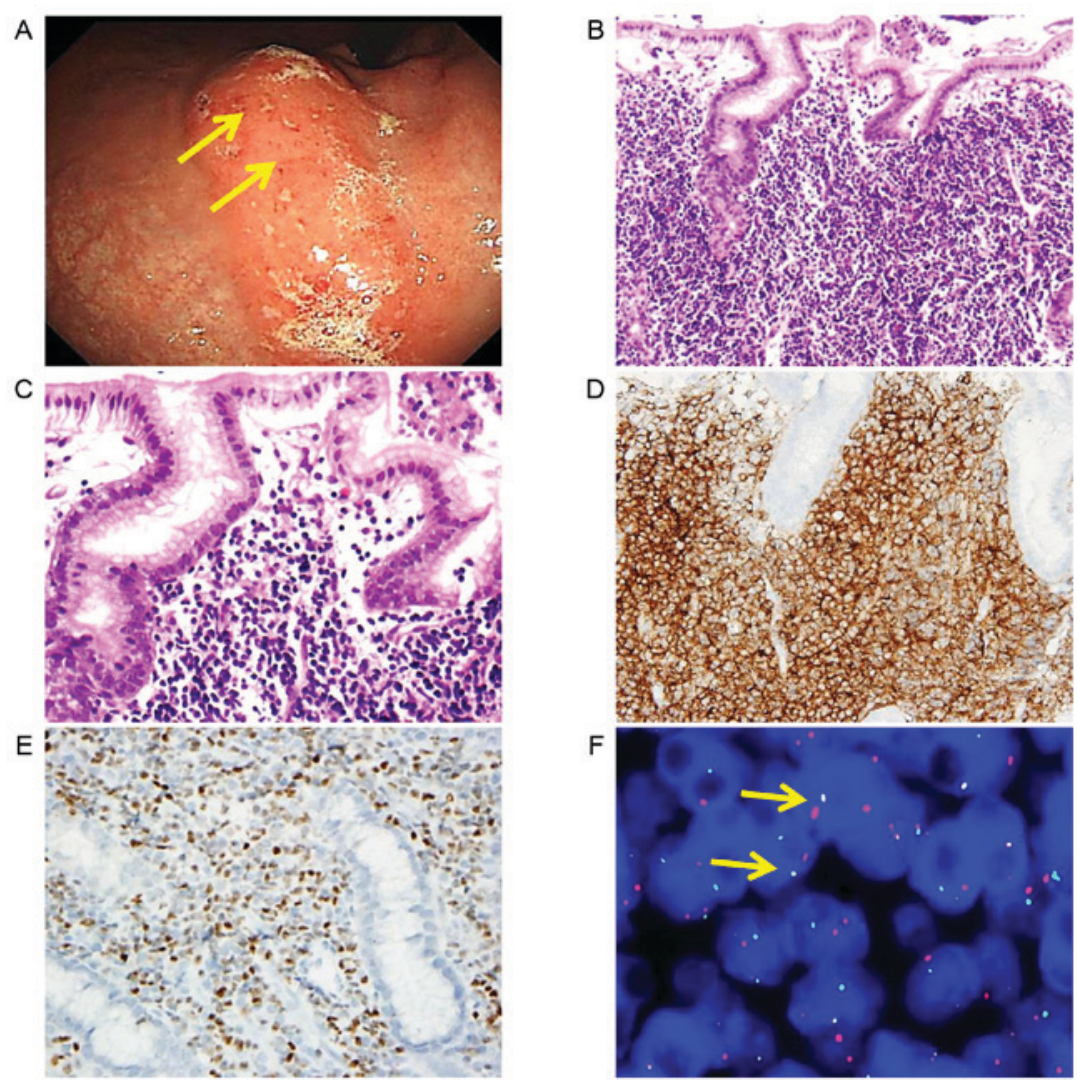

Figure 3. Case 3 was suspected to be a secondary involvement of nodal follicular lymphoma. (A) An endoscopy revealed multiple protruded lesions with erosions. Microscopically, tumor cells diffusely infiltrated the mucosa, without lymphoepithelial lesions, as stained by hematoxylin and eosin and imaged at (B) x200, and (C) x400 magnification. Tumor cells were positive for (D) cluster of differentiation 10 and (E) BCL6 (magnification, x400). (F) immunoglobulin heavy locus/BCL2 translocation was revealed by fluorescence in situ hybridization study using a dual fusion probe. BCL2, B-cell lymphoma 2.

growth advantage to FL cells by interacting with tumor cells. The hollow pattern may be associated with relatively lower histologic grade and the indolent clinical nature of duodenal FL (15). In contrast, case 1 of the present study exhibited preserved FDC nodules, while case 2 revealed near complete loss of FDCs without peripheral accentuation; these patterns differ from the typical hollow pattern of duodenal FL previously reported (13-15). Case 3 also exhibited an absence of an FDC network, similar to case 2. Therefore, the 'hollow pattern' does not appear to be specific to primary gastric FL. Rather, a preserved FDC network may be interpreted as a phenomenon associated with disease progression.

The majority of reported primary gastric FL cases presented with a localized extent of disease. In the present case report, defining the pathological staging of case 1 was difficult due to a concomitant omental tumor nodule without regional lymph node involvement. According to the Tumor-Node-Metastasis staging of the American Joint Committee on Cancer, an omental nodule may be interpreted as a regional lymph node metastasis (N1) or a subserosal invasion (T3) (26). However, there was little evidence of extrafollicular invasion, as observed in the CD10 IHC results in the gastric and omental lesions. Therefore, the omental tumor may be interpreted as multiplicity rather than as metastatic deposit. Multifocality is one of the well-known characteristics in duodenal FL (27). It was concluded that case 3 of the present study was most likely a secondary involvement of the stomach based on Dawson's criteria of primary GI lymphoma (28), and the final stage was designated stage IV due to bone marrow involvement. Unlike primary gastric FLs, secondary gastric involvement may occur in the advanced stage of nodal FL.

Certain studies have identified the lack of BCL2 translocation in primary cutaneous FL as a distinctive feature that differentiates it from nodal FL, suggesting distinct tumorigenic processes in extranodal sites (29). This conclusion was not supported in GI FL; a number of previous studies have identified BCL2 rearrangement in primary intestinal FL $(12,23)$. However, the incidence of BCL2 translocation in primary gastric FL was decreased compared with that in nodal FL, or even duodenal FL, although the expression of BCL2 protein was consistently observed (2). The 2 primary gastric FL cases described in the present study were negative for BCL2 translocation, whereas the case of secondary involvement of nodal FL exhibited translocation $\mathrm{t}(14 ; 18)$. It is possible that the absence of translocation $\mathrm{t}(14 ; 18)$ may serve as a diagnostic indicator, differentiating primary gastric FL from nodal FL. However, this decreased tendency for BCL2 translocation may be as a result of the under-recognition of FL disguised as MALT lymphoma. Nevertheless, translocation-negative cases also revealed an overexpression of BCL2 protein, similar to previous studies, suggesting an alternative genetic mechanism rather than an IGH/BCL2 rearrangement (30).

To the best of our knowledge, there has been no proven etiological agent for gastric FL identified to date, although a number of studies presume that local immunity will contribute to the pathogenesis of FL of the GI tract, just as $H$. pylori participates in the development of MALT lymphoma (31). Local 
Table I. Summarized clinicopathological parameters of cases of gastric follicular lymphoma in the literature.

\begin{tabular}{|c|c|c|c|c|c|c|c|c|c|c|}
\hline $\begin{array}{l}\text { First author, } \\
\text { year }\end{array}$ & $\begin{array}{l}\text { Age, } \\
\text { years }\end{array}$ & Sex & Multiplicity & Grade & $\mathrm{t}(14: 18)$ & H. pylori & Stage ${ }^{a}$ & Treatment & $\begin{array}{c}\text { Outcome, } \\
\text { months }\end{array}$ & (Refs.) \\
\hline \multirow{3}{*}{ Present case } & 61 & M & No & $1-2$ & No & Yes & I & R-CVP & NED, 8 & \\
\hline & 50 & M & No & $1-2$ & No & No & I & Surgery only & NED, 30 & \\
\hline & 40 & M & Yes & $3^{\mathrm{a}}$ & Yes & Yes & IV & R-CVP & PR, 6 & \\
\hline $\begin{array}{l}\text { Takata } \\
\text { et al, } 2013\end{array}$ & $51-81$ & $\begin{array}{l}M(n=4), \\
F(n=4)\end{array}$ & Unknown & $1-2$ & $3 / 8$ & Unknown & $\begin{array}{l}\text { I }(n=5), \\
\text { II }(n=2), \\
\text { IV }(n=1)\end{array}$ & Unknown & Unknown & (9) \\
\hline
\end{tabular}

\begin{tabular}{|c|c|c|c|c|c|c|c|c|c|}
\hline $\begin{array}{l}\text { Tzankov } \\
\text { et al, } 2002\end{array}$ & 72 & F & No & $1-2$ & Yes & Unknown & I & $\mathrm{CHOP}$ & NED, 13 \\
\hline $\begin{array}{l}\text { Huang } \\
\text { et al, } 2008\end{array}$ & 48 & $\mathrm{~F}$ & No & $3^{\mathrm{a}, \mathrm{b}}$ & Unknown & Unknown & IIE & CEOP & NED, 70 \\
\hline $\begin{array}{l}\text { Kanda } \\
\text { et al, } 2003\end{array}$ & 57 & F & Yes & $1-2$ & Yes & Unknown & I & Surgery only & NED, 15 \\
\hline $\begin{array}{l}\text { Iwamuro } \\
\text { et al, } 2013\end{array}$ & 73 & M & No & 1 & No & Yes & IIE & $\begin{array}{l}\text { Surgery and } \\
\text { R-CHOP }\end{array}$ & NED, 30 \\
\hline $\begin{array}{l}\text { Misdragi } \\
\text { et al, } 2001\end{array}$ & 67 & F & Yes & $1-2$ & No & No & I & Rituximab & MOC, 48 \\
\hline
\end{tabular}

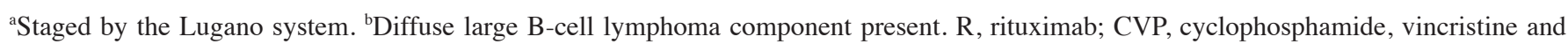
prednisolone; CEOP, cyclophosphamide, epirubicin, vincristine and prednisolone; CHOP, cyclophosphamide, doxorubicin, oncovin and prednisone; NED, no evidence of disease; PR, partial remission; MOC, mortality from other causes; H. pylori, Helicobacter pylori.

antigen-experienced B-cells are suggested to be the origin of primary FL of the GI tract based on the frequent expression of $\operatorname{IgA}$ and the $\alpha 4 \beta 7$ mucosal homing receptor in the tumor cells (32). Colonization of $H$. pylori was identified in case 1 and 3 of the present case report, and Hatano et al (17) revealed an $\sim 50 \%$ incidence of $H$. pylori in gastric FLs in patients of Japanese origin. Considering that the seropositivity for $H$. pylori is $\sim 54 \%$ in patients of Korean origin (33), the causal association of $H$. pylori in gastric FL is not robust enough to be conclusive. However, in case 1 of the present study, follicular gastritis was present in the background mucosa, suggesting the involvement of H. pylori in the tumorigenesis of gastric FL. Although antibiotics targeting $H$. pylori in FL failed in a considerable proportion of patients, there were also a limited number of patients that achieved complete remission (34). Nevertheless, there may be a subgroup of gastric FL associated with a local antigen-presenting environment such as $H$. pylori infection, however, further studies are required to elucidate such cases.

Although the vast majority of GI FLs demonstrate a limited disease extent at the time of diagnosis unlike nodal FLs, the prognosis of gastric FLs does not appear to differ from that of nodal FLs considering the relatively indolent behavior of nodal FLs, even in advanced stages reported in previous studies $(1,2,10,16,24,25,35)$. Based on a review of gastric FLs, including 2 cases from the present study, no disease relapses or disease-associated mortalities have been reported $(13,16,23-25)$. Due to this indolent behavior, despite heterogeneous treatment modalities, therapeutic interventions are recommended only in symptomatic or progressive cases (36). In contrast to the majority of nodal FLs that acquire additional genetic alterations leading to widespread disease and high-grade transformation, FL cells of the GI tract tend to reside in the primary site, potentially explaining the indolent nature of the tumor. There are several reports of gastric FLs with a co-existing diffuse large B-cell lymphoma component, yet such patients generally had favorable disease courses $(23,37)$.

The main differential diagnosis of FL in the GI tract includes florid lymphoid hyperplasia, MALT lymphoma, mantle cell lymphoma and secondary involvement of nodal FL. A distinction from reactive lymphoid hyperplasia may be made in the same context as nodal lesions, where monomorphic composition, absence of tangible body macrophages and architectural disruption favor FL. Occasionally, MALT lymphoma or mantle cell lymphoma is dominated by small lymphocytes with a nodular architecture. Tumor cells in MALT lymphoma present a characteristic lymphoepithelial lesion, which is uncommon in the majority of FL cases $(2,10,23,27,36)$. However, there has been one study of gastric FL with a lymphoepithelial lesion resembling MALT lymphoma (24). Furthermore, certain cases of gastric FL with diffuse mucosal involvement lacking BCL translocation may easily be misinterpreted as MALT lymphoma, particularly in small biopsy specimens (38). Under these circumstances, IHC results usually provide clear distinctions between these types of tumor cell. Neoplastic cells in MALT lymphoma present with negative BCL6 and CD10 IHC test results. As for mantle cell lymphoma, CD5 and cyclin D1 may assist in forming the correct diagnosis. For determining 
the secondary involvement of nodal FL, Dawson's criteria for primary GI lymphoma should be applied (1).

In conclusion, primary gastric FL is a rare, under-recognized disease. Although the general prognosis is indolent, similar to that of nodal FL, a lack of BCL2 rearrangement, low-grade histology with a follicular pattern and sparse mucosal involvement may serve as diagnostic indicators for excluding secondary involvement by nodal FL.

\section{Acknowledgements}

Not applicable.

\section{Funding}

This study was supported by a grant from the Basic Science Research Program through the National Research Foundation of Korea funded by the Ministry of Education (no. 2017R1A2B4005052).

\section{Availability of data and materials}

The datasets used and/or analyzed during the current study are available from the corresponding author on reasonable request.

\section{Authors' contributions}

Conception and design, JK, YK, JC and SM. Administrative support, JK, YK and JH. Provision of clinical data including patient history, endoscopic findings and images of case 1, JS, $\mathrm{SM}, \mathrm{JC}$ and JH. Provision and interpretation of histopathologic, immunohistochemical and molecular analysis of case 1, JS, SM, JC and JH. Provision of clinical data including patient history, endoscopic findings and images of case 2, YK, HK, CL and SS. Provision and interpretation of histopathologic, immunohistochemical and molecular analysis of case 2, YK, HK, $\mathrm{CL}$ and SS. Provision of clinical data including patient history, endoscopic findings and images of case 3, CL, SS and HK. Provision and interpretation of histopathologic, immunohistochemical and molecular analysis of case 3, CL, SS and HK. Collection and assembly of data, HN and YK. Reviewing the slides for histopathological and immunohistochemical features, HN, YK and JK. Reviewing molecular analysis of cases, JH and HK. Reviewing literature, HN, CL and SS. Manuscript writing and revising, $\mathrm{HN}$ and $\mathrm{YK}$. Adding valuable scientific comments in the discussion section, JC, SM, HK and JH. All authors reviewed the manuscript and approved the final article.

\section{Ethics approval and consent to participate}

This study has been granted an exemption from requiring ethics approval by the Institutional Review Board of Seoul National University Boramae Hospital (IRB number, 10-2018-15).

\section{Consent for publication}

Patients' written consent was waived with removal of all identifying information in this retrospective case study under the approval of the Institutional Review Board of Seoul National University Boramae Hospital.

\section{Competing interests}

The authors declare that they have no competing interests.

\section{References}

1. Ghimire P, Wu GY and Zhu L: Primary gastrointestinal lymphoma. World J Gastroenterol 17: 697-707, 2011.

2. Yoshino T, Miyake K, Ichimura K, Mannami T, Ohara N, Hamazaki S and Akagi T: Increased incidence of follicular lymphoma in the duodenum. Am J Surg Pathol 24: 688-693, 2000.

3. Teras LR, DeSantis CE, Cerhan JR, Morton LM, Jemal A and Flowers CR: 2016 US lymphoid malignancy statistics by World Health Organization subtypes. Ca Cancer J Clin: Sep 12, 2016 (Epub ahead of print).

4. Smith A, Crouch S, Lax S, Li J, Painter D, Howell D, Patmore R, Jack A and Roman E: Lymphoma incidence, survival and prevalence 2004-2014: sub-type analyses from the UK's haematological malignancy research network. Br J Cancer 112: 1575-1584, 2015.

5. Lee H, Park HJ, Park E, Ju HY, Oh CM, Kong HJ, Jung KW, Park BK, Lee E, Eom HS and Won YJ: Nationwide statistical analysis of lymphoid malignancies in Korea. Cancer Res Treat 50: 222-238, 2018.

6. Chihara D, Ito H, Matsuda T, Shibata A, Katsumi A, Nakamura S, Tomotaka S, Morton LM, Weisenburger DD and Matsuo K: Differences in incidence and trends of haematological malignancies in Japan and the United States. Br J Haematol 164: 536-545, 2014.

7. Gross SA, Zhu X, Bao L, Ryder J, Le A, Chen Y, Wang XQ and Irons RD: A prospective study of 728 cases of non-Hodgkin lymphoma from a single laboratory in Shanghai, China. Int J Hematol 88: 165-173, 2008.

8. Kim JM, Ko YH, Lee SS, Huh J, Kang CS, Kim CW, Kang YK, Go JH, Kim MK, Kim WS, et al: WHO Classification of malignant lymphomas in Korea: Report of the third nationwide study. J Pathol Transl Med 45: 254-260, 2011.

9. Takata K, Miyata-Takara T, Sato Y and Yoshino T: Pathology of follicular lymphoma. J Clin Exp Hematop 54: 3-9, 2014.

10. Sweldlow SH, Campo E, Harris NL, Jaffe ES, Pileri SA, Stein $\mathrm{H}$ and Thiele $\mathrm{J}$ (eds): WHO Classification of Tumours of Haematopoietic and Lymphoid Tissues, Revised 4th edition, volume 2, IARC Lyon, pp266-273, 2017.

11. Dreyling M, Ghielmini M, Rule S, Salles G, Vitolo U and Ladetto M; ESMO Guidelines Committee: Newly diagnosed and relapsed follicular lymphoma: ESMO clinical practice guidelines for diagnosis, treatment and follow-up. Ann Oncol 27 (Suppl 5): v83-v90, 2016.

12. Goodlad JR, MacPherson S, Jackson R, Batstone P and White J; Scotland and Newcastle Lymphoma Group: Extranodal follicular lymphoma: A clinicopathological and genetic analysis of 15 cases arising at non-cutaneous extranodal sites. Histopathology 44: 268-276, 2004.

13. Takata K, Sato Y, Nakamura N, Tokunaka M, Miki Y, Yukie Kikuti Y, Igarashi K, Ito E, Harigae H, Kato S, et al: Duodenal follicular lymphoma lacks AID but expresses BACH2 and has memory B-cell characteristics. Mod Pathol 26: 22-31, 2013.

14. Takata K, Sato Y, Nakamura N, Kikuti YY, Ichimura K, Tanaka T, Morito T, Tamura M, Oka T, Kondo E, et al: Duodenal and nodal follicular lymphomas are distinct: The former lacks activation-induced cytidine deaminase and follicular dendritic cells despite ongoing somatic hypermutations. Mod Pathol 22: 940-949, 2009.

15. Takata K, Okada H, Ohmiya N, Nakamura S, Kitadai Y, Tari A, Akamatsu T, Kawai H, Tanaka S, Araki H, et al: Primary gastrointestinal follicular lymphoma involving the duodenal second portion is a distinct entity: A multicenter, retrospective analysis in Japan. Cancer Sci 102: 1532-1536, 2011.

16. Kanda M, Ohshima K, Suzumiya J, Haraoka S, Kawasaki C, Sakisaka S and Kikuchi M: Follicular lymphoma of the stomach: Immunohistochemical and molecular genetic studies. J Gastroenterol 38: 584-587, 2003.

17. Hatano B, Ohshima K, Tsuchiya T, Yamaguchi T, Kawasaki C and Kikuchi M: Clinicopathological features of gastric B-cell lymphoma: A series of 317 cases. Pathol Int 52: 677-682, 2002.

18. Choe JY, Yun JY, Na HY, Huh J, Shin SJ, Kim HJ, Paik JH, Kim YA, Nam SJ, Jeon YK, et al: MYC overexpression correlates with MYC amplification or translocation, and is associated with poor prognosis in mantle cell lymphoma. Histopathology 68: 442-449, 2016. 
19. Carbone PP, Kaplan HS, Musshoff K, Smithers DW and Tubiana M: Report of the committee on Hodgkin's disease staging classification. Cancer Res 31: 1860-1861, 1971.

20. Radaszkiewicz T, Dragosics B and Bauer P: Gastrointestinal malignant lymphomas of the mucosa-associated lymphoid tissue: Factors relevant to prognosis. Gastroenterology 102: 1628-1638, 1992.

21. Rohatiner A, d'Amore F, Coiffier B, Crowther D, Gospodarowicz M, Isaacson P, Lister TA, Norton A, Salem P, Shipp M, et al: Report on a workshop convened to discuss the pathological and staging classifications of gastrointestinal tract lymphoma. Ann Oncol 5: 397-400, 1994.

22. Herrmann R, Panahon AM, Barcos MP, Walsh D and Stutzman L: Gastrointestinal involvement in non-Hodgkin's lymphoma. Cancer 46: 215-222, 1980.

23. Huang WT, Hsu YH, Yang SF and Chuang SS: Primary gastrointestinal follicular lymphoma: A clinicopathologic study of 13 cases from Taiwan. J Clin Gastroenterol 42: 997-1002, 2008.

24. Tzankov A, Hittmair A, Müller-Hermelink HK, Rüdiger T and Dirnhofer S: Primary gastric follicular lymphoma with parafollicular monocytoid B-cells and lymphoepithelial lesions, mimicking extranodal marginal zone lymphoma of MALT. Virchows Arch 441: 614-617, 2002.

25. Iwamuro M, Imagawa A, Kobayashi N, Kubota Y, Miyatani K, Takata K and Okada H: Synchronous adenocarcinoma and follicular lymphoma of the stomach. Intern Med 52: 907-912, 2013.

26. Edge S, Byrd D, Compton C, Fritz A, Greene F and Trotti A (eds): AJCC cancer staging manual. Springer, New York, 2010.

27. Misdraji J, Harris NL, Hasserjian RP, Lauwers GY and Ferry JA Primary follicular lymphoma of the gastrointestinal tract. Am J Surg Pathol 35: 1255-1263, 2011.

28. Dawson IM, Cornes JS and Morson BC: Primary malignant lymphoid tumours of the intestinal tract. Report of 37 cases with a study of factors influencing prognosis. Br J Surg 49: 80-89, 1961.

29. Goodlad JR, Krajewski AS, Batstone PJ, McKay P, White JM, Benton EC, Kavanagh GM and Lucraft HH; Scotland and Newcastle Lymphoma Group: Primary cutaneous follicular lymphoma: A clinicopathologic and molecular study of 16 cases in support of a distinct entity. Am J Surg Pathol 26: 733-741, 2002.

30. Horsman DE, Okamoto I, Ludkovski O, Le N, Harder L, Gesk S, Siebert R, Chhanabhai M, Sehn L, Connors JM and Gascoyne RD: Follicular lymphoma lacking the $\mathrm{t}(14 ; 18)(\mathrm{q} 32 ; \mathrm{q} 21)$ : identification of two disease subtypes. Br J Haematol 120: 424-433, 2003.
31. Hussell T, Isaacson PG, Crabtree JE and Spencer J: Helicobacter pylori-specific tumour-infiltrating $\mathrm{T}$ cells provide contact dependent help for the growth of malignant B cells in low-grade gastric lymphoma of mucosa-associated lymphoid tissue. J Pathol 178: 122-127, 1996.

32. Bende RJ, Smit LA, Bossenbroek JG, Aarts WM, Spaargaren M, de Leval L, Boeckxstaens GE, Pals ST and van Noesel CJ: Primary follicular lymphoma of the small intestine: Alpha4beta7 expression and immunoglobulin configuration suggest an origin from local antigen-experienced B cells. Am J Pathol 162: 105-113, 2003.

33. Lim SH, Kwon JW, Kim N, Kim GH, Kang JM, Park MJ, Yim JY, Kim HU, Baik GH, Seo GS, et al: Prevalence and risk factors of Helicobacter pylori infection in Korea: Nationwide multicenter study over 13 years. BMC Gastroenterol 13: 104, 2013.

34. Nakamura S, Matsumoto T, Umeno J, Yanai S, Shono Y, Suekane H, Hirahashi M, Yao T and Iida M: Endoscopic features of intestinal follicular lymphoma: The value of double-balloon enteroscopy. Endoscopy 39 (Suppl 1): E26-E27, 2007.

35. Luminari S, Bellei M, Biasoli I and Federico M: Follicular lymphoma-treatment and prognostic factors. Rev Bras Hematol Hemoter 34: 54-59, 2012.

36. Schmatz AI, Streubel B, Kretschmer-Chott E, Püspök A, Jäger U, Mannhalter C, Tiemann M, Ott G, Fischbach W, Herzog P, et al: Primary follicular lymphoma of the duodenum is a distinct mucosal/submucosal variant of follicular lymphoma: A retrospective study of 63 cases. J Clin Oncol 29: 1445-1451, 2011.

37. Maeshima AM, Omatsu M, Nomoto J, Maruyama D, Kim SW, Watanabe T, Kobayashi Y, Tobinai K and Matsuno Y: Diffuse large B-cell lymphoma after transformation from low-grade follicular lymphoma: Morphological, immunohistochemical, and FISH analyses. Cancer Sci 99: 1760-1768, 2008.

38. Burke JS: Lymphoproliferative disorders of the gastrointestinal tract: A review and pragmatic guide to diagnosis. Arch Pathol Lab Med 135: 1283-1297, 2011

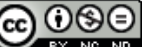

This work is licensed under a Creative Commons Attribution-NonCommercial-NoDerivatives 4.0 International (CC BY-NC-ND 4.0) License. 